\title{
Balloon-Borne Experiment for Deep Sky Survey of MeV Gamma Rays using an Electron-Tracking Compton Camera
}

\author{
Shotaro Komura ${ }^{* a}$, Toru Tanimori ${ }^{a}$, Atsushi Takada ${ }^{a}$, Hidetoshi Kubo ${ }^{a}$, Satoru \\ Iwaki $^{a}$, Yoshihiro Matsuoka ${ }^{a}$, Tetsuya Mizumoto ${ }^{a}$, Yoshitaka Mizumura ${ }^{a}$, Tetsuro \\ Kishimoto $^{a}$, Taito Takemura ${ }^{a}$, Shohei Miyamoto ${ }^{a}$, Kiseki Nakamura ${ }^{a}$, Shogo \\ Nakamura $^{a}$, Makoto Oda ${ }^{a}$, Shinya Sonoda ${ }^{a}$, Dai Tomono ${ }^{a}$, Joseph Don Parker ${ }^{a}$, \\ Kentaro Miuchi $^{b}$, Tatsuya Sawano ${ }^{c}$, Shunsuke Kurosawa ${ }^{d}$ \\ ${ }^{a}$ Kyoto University \\ ${ }^{b}$ Kobe University \\ ${ }^{c}$ Kanazawa University \\ ${ }^{d}$ Tohoku University \\ E-mail: komura@cr.scphys.kyoto-u.ac.jp
}

Despite the scientific importance of $\mathrm{MeV}$ gamma-ray studies, sufficient observations have not been performed due to the large radiation backgrounds and the unclearness of MeV gamma-ray imaging. To advance the MeV gamma-ray astronomy, we have developed an Electron-Tracking Compton Camera (ETCC) with a gaseous electron tracker. By measuring three dimensional tracks of Compton-recoil electrons, our ETCC has attained the high-quality imaging and powerful background rejection. In order to verify such performance of an ETCC, we have carried out the balloon-borne experiments, "Sub-MeV gamma-ray Imaging Loaded-on-balloon Experiment" (SMILE) since 2006. The performance of current ETCC has already been surpassed the requirements to detect the Crab Nebula for 5 sigma level with several hours balloon observations. In addition, the ability of the polarization measurements has been revealed. The modulation factor is estimated to be 0.6 for the energy region below $200 \mathrm{keV}$ by the Monte Carlo simulation. We have measured the polarization ability using the polarized X-ray beam-line at SPring- 8 , and then modulation factor of 0.6 is obtained at $130 \mathrm{keV}$, which is consistent with the results of the simulation and shows that the ETCC has an excellent performance as a sub-MeV gamma-ray polarimeter. By using the pressured $\mathrm{CF}_{4}$ based gas at $3 \mathrm{~atm}$, the detection efficiency of the ETCC will be increased one order. Therefore we have a plan of the long duration observation for deep sky survey with polarization measurements of bright sources including Gamma-Ray Bursts. Here we present the concept of ETCC and the future prospects based on the performance of the current ETCC.

The 34th International Cosmic Ray Conference,

30 July- 6 August, 2015

The Hague, The Netherlands

\footnotetext{
*Speaker.
} 


\section{Introduction}

Sub-MeV/MeV gamma-ray observations are a key to understand various high-energy phenomena. The nuclear gamma-ray lines, mainly emitted in $\mathrm{MeV}$ energy region, are known as examples of the unique probe to investigate the nucleosynthesis process. As for the polarization measurement, it enable us to estimate the magnetic field configuration on the non-thermal radiation process directly. Despite such the scientific importance of $\mathrm{MeV}$ gamma-ray studies, sufficient observations have not been done in past gamma-ray missions. In the energy range from 0.75 to $30 \mathrm{MeV}$, approximately only 30 persistent gamma-ray sources have been reported by the Imaging Compton Telescope (COMPTEL) in 10 years all-sky survey [1], while Fermi-LAT has found nearly 3000 sources in the energy region from 0.1 to $100 \mathrm{GeV}$ [2]. There has been a few polarization measurement. INTEGRAL satellite reported the polarization with the large error of two brightest MeV gamma-ray sources, Crab Nebula and Cygnus X-1 in the 0.1 to $2 \mathrm{MeV}$ band [3] [4] [5]. As to transient objects, Gamma-ray Burst Polarimeter (GAP) detected the polarization of the 3 bright Gamma-Ray Bursts (GRB) at 3 sigma level in the 80 to $300 \mathrm{keV}$ band during two-year observations [6] [7].

The detection sensitivity of $\mathrm{MeV}$ gamma ray in space is primarily limited by the huge backgrounds produced in the interaction of cosmic rays with instruments and the unclearness of $\mathrm{MeV}$ gamma-ray imaging. This is the reason why COMPTEL operated with only one-third of the sensitivity expected from the ground calibration tests [8]. Then Schönfelder (a leader of COMPTEL team) pointed out that localising the arrival direction on the Compton event circle by measuring the direction of the Compton-recoil electron, which means improving the point spread function (PSF), is a very significant background reduction [9].

Then, we have developed an Electron-Tracking Compton Camera (ETCC) having powerful background suppression tools and capabilities to achieve a good PSF by measuring threedimensional track of Compton-recoil electron. In order to verify the performance of an ETCC in space, we started the balloon experiments " Sub-MeV gamma-ray Imaging Loaded-on-balloon Experiment" (SMILE) since 2006 for the future satellite observation with several ten times better sensitivities than that of COMPTEL. In Section 2, we introduce the concept of ETCC; in Section 3, after the brief description of the current ETCC about the design and performance for gamma-ray detection, we report on the latest results about the polarization measurement; finally, we discuss the results and the future prospects for SMILE project in Section 4.

\section{Concept of ETCC}

\subsection{Compton reconstruction and Imaging}

An ETCC consists of a gaseous time projection chamber (TPC) as a Compton scatterer and pixel scintillator arrays (PSAs) as an absorber for Compton-scattered gamma ray. The left panel of figure 1 shows the schematic view of the current ETCC described in detail in Section 3 TPC is based on a two-dimensional micro pattern gas detector with 400 um pitch pixels to measure the fine three-dimensional tracks and energy deposit of charged particles. The right two panels of figure 1 are examples of two-dimensional projected track images obtained by a $30 \mathrm{~cm}$-cubic TPC. The position of Compton scattering and the initial direction of the Compton-recoil electron are obtained by track fitting. The absorption position of Compton-scattered gamma ray and its 

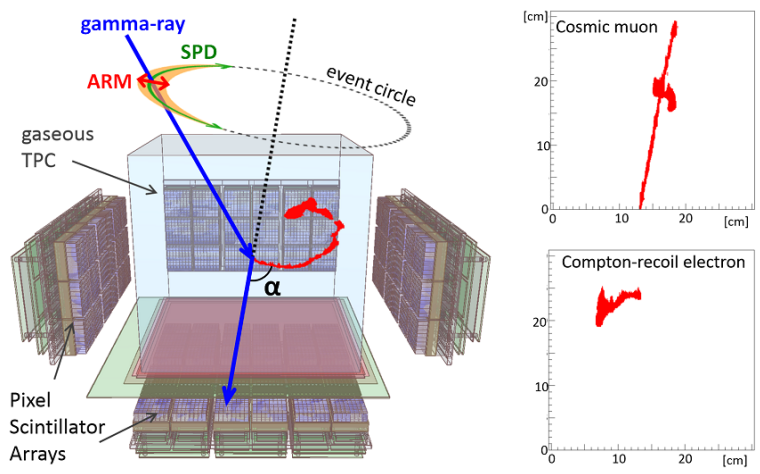

Figure 1: Left: Schematic view of ETCC which consists of a gaseous electron tracker surrounded by PSAs. Right: Projected track images of charged particles obtained by $30 \mathrm{~cm}$-cubic TPC (top panel shows cosmic muon with delta-ray, bottom panel shows Compton-recoil electron).

energy are measured by PSAs. An ETCC measures all of the parameters of the Compton scattering kinematics, and then we can reconstruct the energy and the arrival direction of the incident gamma ray event by event.

For conventional Compton cameras like COMPTEL, without electron track information, the arrival direction of the incident gamma ray is reconstructed with a doughnut-shaped uncertainty. Measurements of electron tracks improve the uncertainty of the direction dramatically. An ETCC reconstructs the direction with a crescent-shape uncertainty characterized by a combination of the resolution of the Compton scattering angle (angular resolution measure, ARM) and directional angular resolution of the Compton scatter plane (scatter plane deviation, SPD). Examples of the overlaying image of several reconstructed gamma rays are shown in figure 2 The conventional Compton image is widely spread, and there appear some artifact peaks which cause a serious decrease in the signal-to-noise ratio. Thus, SPD obviously improve the PSF and better PSF certainly suppresses the background by restricting the source region. The PSF of ETCC is estimated with only the resolutions of ARM and SPD without the use of optimization algorithm as like maximum likelihood-expectation maximization. Therefore, the sensitivity should be determined simply from the effective area and the PSF. The details are discussed in [11], and it shows the reasonable expectation that the sensitivity of ETCC will reach to $1 \mathrm{mCrab}$ in the near future.

\subsection{Background rejection}

The information of an electron track also gives us the tools to suppress various backgrounds. The angle $\alpha$ (see figure 1) is obtained by two independent ways. One is geometrically, calculating the inner product of the recoil electron vector and the scattered gamma-ray vector. The other is kinetically, calculating based on Compton kinematics with the measured energy deposit in TPC and PSAs. By checking the consistency between these two values, an ETCC rejects Compton scattering fake events such as chance coincidence hits of the TPC and PSAs and events where Compton scattering occurred in the PSAs.

We can also obtain the energy-loss rate $(\mathrm{dE} / \mathrm{dx})$ of charged particles interacted in TPC by using the relationship of its energy and range calculated from the track information. Therefore, we can 

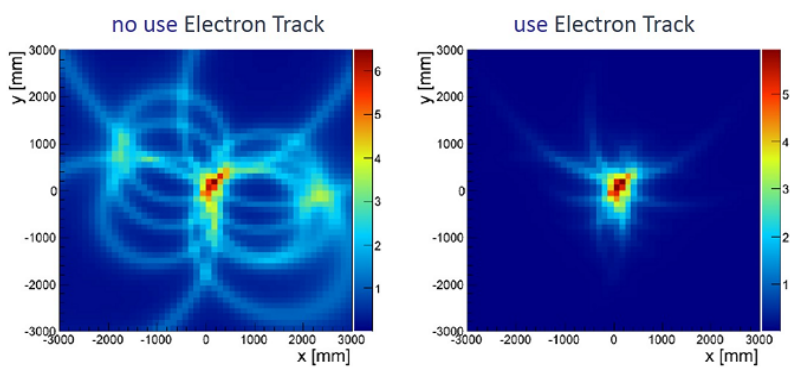

Figure 2: Comparison of the two different imaging technique, whether or not using the information of electron track. The left panel is the COMPTEL-type conventional imaging, where the reconstructed gamma rays are drawn as doughnut-shapes. The right panel is the ETCC-type imaging, where the gamma rays are drawn as crescent-shapes. These are adapted from [10].

identify the kind of particles and reject non gamma-ray backgrounds, such as cosmic rays, neutrons and Compton-recoil electrons escaping from TPC. Thanks to these background suppression tools, without an active veto shielding, ETCC also has the large Field of View (FoV).

\subsection{Polarization measurement}

In Compton scattering, polarized gamma rays tend to scatter perpendicularly at the scattering plane, which causes the modulated distribution of the scattered azimuthal angle $\phi$, according to the Compton scattering cross-section described by the Klein-Nishina formula. A Compton camera measures the three-dimensional scattering direction of incident gamma rays, therefore it can also be operated as a polarimeter.

The modulation curve is drawn as a function of $\cos 2 \phi$ theoretically. The modulation factor (MF) is an instrumental performance parameter of polarization measurements, which is defined as the ratio of the amplitude to the average level of the modulation curve for $100 \%$ linearly polarized incident gamma rays. If the MF is obtained, we can calculate the minimum detectable polarization (MDP), a polarimetric sensitivity of the detector, from the effective area and estimated signal and background rates [12]. Since for space observations the background rate is much higher than the signal rate, the MDP deteriorate rapidly in proportion to the square root of the background rate. Thus, ETCC has a large advantage to keep the MDP even in the intense background condition in that the backgrounds are suppressed by high-quality imaging ability based on SPD and background rejection tools as mentioned above. In addition, the large FoV of ETCC is very efficient characteristic not only to increase the observation time for persistent sources, but also to provide us the chance of detection for transient objects including GRBs.

\section{Status of current ETCC}

\subsection{Instrument}

We had the first balloon experiment SMILE-I in 2006 using a small size ETCC with $10 \mathrm{~cm}$ cubic TPC and succeeded to observe the diffuse cosmic and atmospheric gamma-ray fluxes as well as to revel the good background rejection ability of the ETCC [13]. As the next step of SMILE 


\begin{tabular}{|c|c|c|}
\hline $\mathrm{TPC}$ & $\begin{array}{c}\text { Volume } \\
\text { Gas } \\
\text { Spatial resolution } \\
\text { Energy resolution (FWHM) }\end{array}$ & $\begin{array}{c}30 \times 30 \times 30 \mathrm{~cm}^{3} \\
\text { Ar:iso- } \mathrm{C}_{4} \mathrm{H}_{10}: \mathrm{CF}_{4}(95: 2: 3) \text { at } 1 \mathrm{~atm} \\
\sim 0.5 \mathrm{~mm} \\
22 \% \text { at } 22 \mathrm{keV}\end{array}$ \\
\hline PSAs & $\begin{array}{c}\text { Scintillator } \\
\text { Pixel size } \\
\text { Number of pixels } \\
\text { Dynamic range } \\
\text { Energy resolution (FWHM) }\end{array}$ & $\begin{array}{c}\text { GSO:Ce }\left(6.71 \mathrm{~g} / \mathrm{cm}^{3}\right) \\
6 \times 6 \times 13 \mathrm{~mm}^{3} \\
6912 \\
80 \mathrm{keV}-1.3 \mathrm{MeV} \\
10 \% \text { at } 662 \mathrm{keV}\end{array}$ \\
\hline
\end{tabular}

Table 1: Specifications of SMILE-II ETCC

(SMILE-II), we plan to observe bright celestial sources such as Crab nebula and Cygnus X-1 to verify the gamma-ray imaging ability of an ETCC. The requirements for 5 sigma level detection with several hours balloon observations are an effective area grater than $0.5 \mathrm{~cm}^{2}$ for energy below $300 \mathrm{keV}$ and ARM resolution less than 10 degrees at $662 \mathrm{keV}$ [14]. In 2013, we developed a medium size ETCC with $30 \mathrm{~cm}$-cubic TPC and 108 PSAs as the flight model detector of SMILE-II [10] [15]. As shown in figure 1, 36 PSAs are placed under the bottom of the TPC, and the other 72 PSAs are set around the four lateral sides of the TPC. The specifications of SMILE-II ETCC are summarized in Table 1

\subsection{Performance for gamma-ray detection}

The performance parameters of SMILE-II ETCC for gamma-ray detection is already measured by the ground-based experiments using several radio isotopes [16]. The effective area is close to $1 \mathrm{~cm}^{2}$ for energy below $300 \mathrm{keV}$, and its energy dependence in 0.15 to $1 \mathrm{MeV}$ energy range are consistent with the results of the Monte Carlo simulation [17] based on the Geant4 tool kit, which shows the reliability of this simulation. The FoV is measured to be $6 \mathrm{sr}(>50 \%$ of the on-axis detection efficiency) for $662 \mathrm{keV}$ incident gamma rays. The ARM resolution at $662 \mathrm{keV}$ is $5.3 \mathrm{de}-$ grees (FWHM) which is close to the limit determined by the energy resolution of PSAs. The SPD resolution for Compton-recoil electrons in $10 \mathrm{keV}$ to $300 \mathrm{keV}$ energy range is approximately 50 to 100 degrees which is near the value expected from the multiple scattering and spatial resolution of the TPC. Thus the performance of SMILE-II ETCC has been surpassed the requirements. We already confirmed that thanks to the background suppression abilities ETCC can keep the sensitivity stably even in the intense radiation condition five times more intense than that encountered at the balloon altitude [18].

\subsection{Performance for polarization measurement}

We studied the ability of the ETCC as a gamma-ray polarimeter by Geant4-based simulation with a detailed geometrical model of SMILE-II ETCC and the physics list named G4EmLivermorePolarizedPhysics including polarized gamma-ray models. For $200 \mathrm{keV}$ gamma rays on axis, two types of the azimuthal angle distribution are obtained whether the gamma rays are non-polarized or $100 \%$ polarized as shown in figure $3 \mathrm{k}$ and $3 \mathrm{~b}$, respectively. Even non-polarized distribution reveals the modulation due to the variation in the detection efficiency derived from the geometrical configuration. Dividing the obtained distribution of $100 \%$ polarized photons by that of non-polarized photons, this geometrical modulation is canceled and the $\cos 2 \phi$ shaped polarimetric modulation 

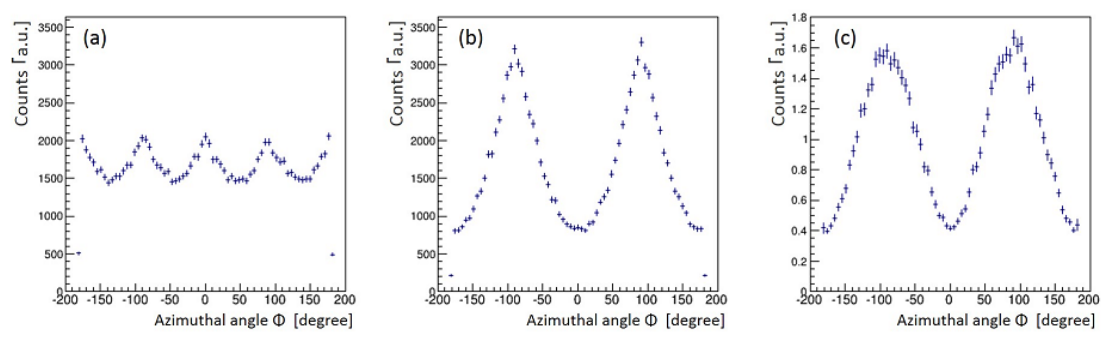

Figure 3: Azimuthal angle distributions of scatted photons for incident on-axis gamma rays with $200 \mathrm{keV}$ energy calculated by the Geant4-based simulation for SMILE-II ETCC: (a) non-polarized incident photons; (b) $100 \%$ polarized incident photons; (c) corrected $100 \%$ polarized distribution.

appears as shown in figure $3 \mathrm{k}$. The calculated MFs from corrected modulation curves are 0.6 for the energy range below $200 \mathrm{keV}$ and 0.5 at $500 \mathrm{keV}$, which indicates that the ETCC has the excellent performance as a sub-MeV gamma-ray polarimeter in a wide energy range.

In order to confirm these results, we have measured the modulation factor of SMILE-II ETCC at High Energy Inelastic Scattering Beamline BL08W of SPring-8. The experimental setup is shown in the figure 4 a. We irradiated a $182 \mathrm{keV}$ X-ray with nearly $100 \%$ linear polarization to an aluminum (Al) target, and scattered photons perpendicularly at the target enter the ETCC. Due to the spatial limit, the $\mathrm{Al}$ target was located at a position close to the ETCC, and the incoming photons had a wide incident angle of plus or minus 21 degrees. Therefore the energy and the degree of polarization was also widely spread from 123 to $148 \mathrm{keV}$ and 0.93 to 0.98 , respectively. The acquisition rate of $\mathrm{Al}$ target-on data was approximately $300 \mathrm{~Hz}$ while that of Al target-off data was about $150 \mathrm{~Hz}$. We selected the valid events using the simple analysis of electron track [10] including the $\mathrm{dE} / \mathrm{dx}$ identification. The figure $4 \mathrm{~b}$ shows the results of the reconstructed energy spectra of valid events. In the energy spectrum of the on-target data after subtracting the off-target energy spectrum, an energy peak is visible in the expected energy range, which shows that ETCC succeeded to detect the scattered gamma-ray event definitely. In order to measure the detector response for the MF to various polarization vectors, the ETCC was mounted on a rotation stage. The measurements were performed in five different rotation angles where the polarization angle $\phi_{p o l} \mathrm{~s}$ were $0,-22.5,-45,-90$ and -180 degrees, respectively. By combining the azimuthal angle distribution of 0 and -90 degrees, we made the response of the non-polarized gamma rays for the cancellation of the geometrical factor. The figure $3 \mathrm{k}$ shows the obtained modulation curves. We can see that ETCC clearly detected the polarization modulations and the phase shifts of modulation curves are consistent with the experimental setup. From these results we obtained the MF of SMILE-II ETCC as 0.6 at $130 \mathrm{keV}$, which matches the results of the simulation mentioned above.

\section{Discussion and future prospects}

We are studying the design of the SMILE-III ETCC to observe lots of celestial sources with a better sensitivity and to measure the polarization of bright sources. Simulations have shown that the effective area will increase to $20 \mathrm{~cm}^{2}$ by using $\mathrm{CF}_{4}$ gas at 3 atm and 3 radiation lengths thick PSAs. Thus we consider the one-month balloon-flight at the polar region for deep sky survey with a several times better sensitivity than COMPTEL. We also estimated the MDPs, considering the simulated 


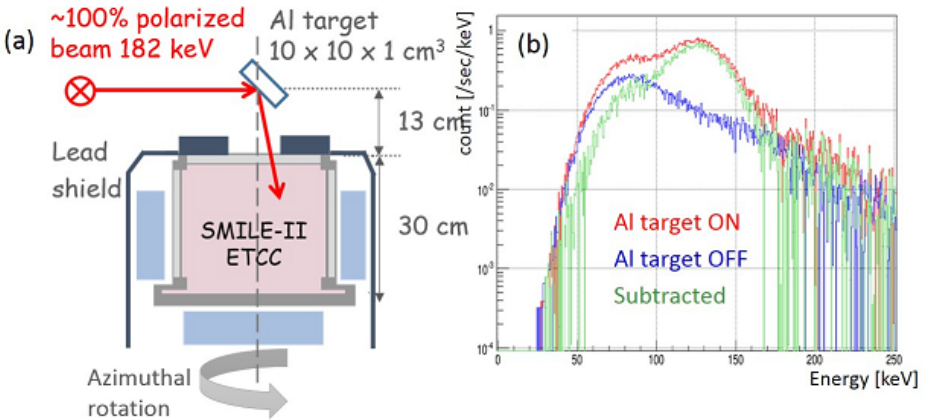

Figure 4: (a) Schematic view of the setup for polarization measurements at BL08W of SPring-8. (b) Reconstructed energy spectra of incident photon. The red and blue lines represent $\mathrm{Al}$ target-on data and $\mathrm{Al}$ target-off data, respectively. The green line is the difference between the red and blue lines, represents the energy spectrum of incident gamma ray scattered at Al target.
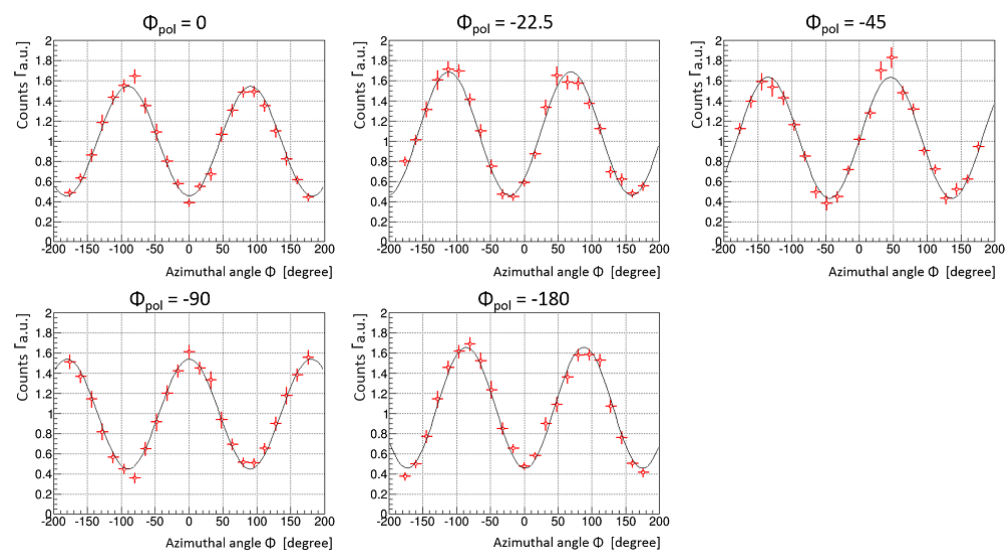

Figure 5: Corrected modulation curves and their fit curves for the five experimental setups with different polarization angles.

MF, calculated effective area of SMILE-III ETCC and the assumed backgrounds. Backgrounds include an extragalactic diffuse gamma ray, an atmospheric gamma ray and an intrinsic gamma ray calculated by simulations based on the results of SMILE-I experiment. The MDPs for 3 sigma level detection of Crab nebula and Cygnus X-1 is approximately 15\% and 20\%, respectively. The degree of polarization of Crab reported by INTEGRAL satellite are around 40\% [3], thus our SMILE-III ETCC would perform better polarimetric observations. Furthermore, SMILE-III ETCC will also detect the polarization of typical GRBs. For one-month flight, a few GRBs with the intensity $10^{-6}$ $\mathrm{erg} / \mathrm{cm}^{2} / \mathrm{s}$ and about $10 \mathrm{GRBs}$ with the intensity $10^{-7} \mathrm{erg} / \mathrm{cm}^{2} / \mathrm{s}$ are expected and the MDPs of them are calculated as approximately $6 \%$ and $20 \%$, respectively.

\section{Acknowledgment}

This study was supported by a Grant-in-Aid for Scientific Research from the Ministry of Education, Culture, Sports, Science and Technology (MEXT) of Japan (Grant numbers 21224005, 20244026, 23654067, 25610042), a Grant-in-Aid from the Global COE program “Next Generation 
Physics, Spun from Universality and Emergence” from the MEXT of Japan, and a Grant-in-Aid for JSPS Fellows (Grant numbers 13J01213). This study was also supported by “SENTAN” program promoted by Japan Science and Technology Agency (JST). The synchrotron radiation experiments were performed at the BL08W of SPring-8 with the approval of the Japan Synchrotron Radiation Research Institute (JASRI) (Proposal No. 2014B1088). Some of the electronics development was supported by KEK-DTP and Open-It.

\section{References}

[1] V. Schönfelder et al., The first COMPTEL source catalogue, A\&AS 143 (2000) 145.

[2] F. Acero et al., Fermi Large Area Telescope Third Source Catalog, ApJS 218 (2015) 23.

[3] A. J. Dean, et al., Polarized Gamma-Ray Emission from the Crab, Science 321 (2008) 1183.

[4] M. Forot, et al., Polarization of the Crab pulsar and nebula as observed by the Integral/IBIS telescope, ApJ 688 (2008) 29.

[5] P. Laurent, et al., Polarized Gamma-Ray Emission from the Galactic Black Hole Cygnus X-1, Science 332 (2011) 438.

[6] D. Yonetoku, et al., Detection of Gamma-Ray Polarization in Prompt Emission of GRB 100826A, ApJ 743 (2011) L30.

[7] D. Yonetoku, et al., Magnetic Structures in Gamma-Ray Burst Jets Probed by Gamma-Ray Polarization, ApJ 758 (2012) L1.

[8] G. Weidenspointner, et al., The COMPTEL instrumental line background, A\&A 368 (2001) 347.

[9] V. Schönfelder, Lessons learnt from COMPTEL for future telescopes, New Astron. Rev. 48 (2004) 193.

[10] Y. Mizumura et al., Development of a $30 \mathrm{~cm}$-cube Electron-Tracking Compton Camera for the SMILE-II Experiment, JINST 9 (2014) C05045.

[11] T. Tanimori et al., An Electron-Tracking Compton Telescope for a Survey of the Deep Universe by MeV gamma-rays, ApJ (2015) accepted, arXiv:1507.03850 [astro-ph.IM].

[12] F. Lei et al., Compton Polarimetry in Gamma-ray Astronomy, Space Sci. Rev. 82 (1997) 309.

[13] A. Takada et al., Observation of Diffuse Cosmic and Atmospheric Gamma Rays At Balloon Altitudes With an Electron-Tracking Compton Camera, ApJ 733 (2011) 13.

[14] A. Takada et al., SMILE-II: Observation of Celestial and Atmospheric MEV Gamma Rays Using a Balloon-Borne Wide Fields of View Electron-Tracking Compton Camera, in proceedings of The 20th Symposium on European Rocket and Balloon Programmes and Related Research, (2011) 567.

[15] T. Mizumoto et al., New readout and data-acquisition system in an electron-tracking Compton camera for MeV gamma-ray astronomy (SMILE-II), NIMA (2015) accepted, arXiv:1508.00990 [astro-ph.IM].

[16] A. Takada et al., MeV gamma-ray Compton camera using a gaseous electron tracker for background-suppressed observation, in proceedings of SPIE, Space Telescopes and Instrumentation 2014 (2014) 91440G.

[17] T. Sawano et al., SMILE-II: Balloon-borne Telescope for Background-suppressed Soft Gamma-ray Imaging, JPS Conference Proceedings 1 (2014) 013099.

[18] Y. Matsuoka et al., Performance of a New Electron-Tracking Compton Camera under Intense Radiations from a Water Target irradiated with a Proton Beam, JINST 10 (2015) C01053. 